\title{
EphA2 chimeric antigen receptor-modified T cells for the immunotherapy of esophageal squamous cell carcinoma
}

\author{
Hui Shi ${ }^{1}$, Feng $\mathbf{Y u}^{2}$, Yinting Mao ${ }^{1}$, Qianqian $\mathrm{Ju}^{1}$, Yingcheng $\mathrm{Wu}^{3}$, Wen Bai ${ }^{3}$, Peiwen Wang ${ }^{3}, \operatorname{Ran} \mathrm{Xu}^{3}$, \\ Maorong Jiang ${ }^{4}$, Jiahai Shi ${ }^{1}$ \\ ${ }^{1}$ Department of Thoracic Surgery, Affiliated Hospital of Nantong University, Nantong 226001, China; ${ }^{2}$ Institute of Life Sciences, Jiangsu University, \\ Zhenjiang 212013, China; ${ }^{3}$ Medical College, ${ }^{4}$ Laboratory Animals Center, Nantong University, Nantong 226001, China \\ Contributions: (I) Conception and design: J Shi, M Jiang, H Shi, F Yu; (II) Administrative support: J Shi; (III) Provision of study materials or patients: \\ None; (IV) Collection and assembly of data: Y Mao, Q Ju, Y Wu, W Bai, P Wang, R Xu; (V) Data analysis and interpretation: J Shi, M Jiang, H Shi, \\ F Yu, Y Mao, Q Ju; (VI) Manuscript writing: All authors; (VII) Final approval of manuscript: All authors. \\ Correspondence to: Jiahai Shi. Department of Thoracic Surgery, Affiliated Hospital of Nantong University, 20 Xisi Road, Nantong 226001, China. \\ Email: nt_shijiahai@163.com; Maorong Jiang. Laboratory Animals Center, Nantong University, 19 Qixiu Road, Nantong 226001, China. \\ Email: jiangmr@ntu.edu.cn.
}

Background: It is urgent to explore an effective potential therapeutic strategy for ESCC. In recent years, cell-based cancer immunotherapy has become a potentially close for carcinoma therapy. Chimeric antigen receptor (CAR) $\mathrm{T}$ cell technology is a kind of adoptive cell therapy technique which has been developed rapidly. We sought to obtain EphA2.CAR-T cell and revealed the ability of EphA2.CAR-T cells to kill esophageal squamous cell carcinoma (ESCC) cells in vitro.

Methods: Firstly, the expression and location of EphA2 in ESCC tissues and cells was tested by immunohistochemistry staining and Western blot. Secondly, the second generation of EphA2.CAR was constructed via molecular biology technology, and transduced into T cells to obtain the EphA2.CAR-T cell. The transduction efficacies were assessed using flow cytometry (FCM). Thirdly, the effect of cell killing of EphA2.CAR-T cell on ESCC cells in vitro was detected by co-culture experiments. The productions of cytokines (TNF- $\alpha$ and IFN- $\gamma$ ) by EphA2.CAR-T cell after co-culture with ESCC cells were analyzed by ELISA assay.

Results: The expression of EphA2 was significantly upregulated in ESCC tissues and cells $(\mathrm{P}<0.05)$. EphA2 was expressed on the membrane of ESCC cells, so it could be served as tumor-associated surface antigens (TAA) of CAR for ESCC treatment. The EphA2.CAR-T cell was obtained successfully, and its' transduction efficacies was $61.4 \%$ by FCM. The ability of cell killing of EphA2.CAR-T cell was better than that of T cells $(\mathrm{P}<0.01)$, and demonstrated a dose-dependent cell killing. The results of ELISA assay showed that the levels of TNF- $\alpha$ and IFN- $\gamma$ in EphA2.CAR-T cells were notably raised compared with T cells $(\mathrm{P}<0.05)$.

Conclusions: We firstly constructed the second generation of EphA2.CAR and established EphA2.CAR-T cells. The EphA2.CAR-T cells showed a dose-dependent cell killing of ESCC cells, and promoted the production of cytokines in vitro. These findings open a new way for treatment of ESCC by immunotherapy in the future.

Keywords: Esophageal squamous cell carcinoma (ESCC); chimeric antigen receptor (CAR); erythropoietinproducing hepatocellular receptor A2 (EphA2); cell killing; TNF- $\alpha$; IFN- $\gamma$

Submitted Nov 28, 2017. Accepted for publication Mar 12, 2018.

doi: $10.21037 /$ jtd.2018.04.91

View this article at: http://dx.doi.org/10.21037/jtd.2018.04.91 


\section{Introduction}

Esophageal cancer is a common digestive tract tumor and a malignant tumor formed by abnormal hyperplasia of esophageal squamous epithelium or glandular epithelium $(1,2)$. Because $80 \%$ of esophageal cancer is composed of esophageal squamous cell carcinoma (ESCC), and based on the improvement of pathological diagnosis and treatment of ESCC, the slight improvement of clinical treatment of ESCC has been promoted. However, the newer and more effective therapies strategies are needed to optimize and promote the clinical treatment of ESCC (3).

Cancer immunotherapy is to kill tumors and inhibit tumor growth by increasing the immunogenicity of tumor cells and the cytotoxicity of effector cells, enhancing immune response for anti-tumor cooperating with the immune system (4-6). T lymphocytes, as the core immune cells that mediate adaptive cellular immune response, could kill tumor cells. In recent years, cell-based cancer immunotherapy has become a potentially close for carcinoma treatment. System modified T cells to express chimeric antigen receptor (CAR), known as CAR T cells, evolve the ordinary $\mathrm{t}$ cells to distinguish specific antigen and target tumor cells $(7,8)$. Therefore CARs modified $\mathrm{T}$ cell therapy is one of cell-based cancer immunotherapy $(9,10)$. CAR-modified T (CAR-T) cells are not only major histocompatibility complex (MHC)-restricted but also can be engineered to specifically recognize tumor-associated surface antigens (TAA) (11). CAR is a fusion structure formed by a simulation of physiological function of $\mathrm{T}$ cell receptor (TCR) (12). Specifically recognition and following signaling cascade is due to the structural characteristics of CAR $(13,14)$. According to the combination of stimulating signal molecules, CAR-T cells were separated into three generations (15). The 2 nd generation CAR-T cells were concerned with the introduction of one co-stimulatory molecule (16-18). The 3rd generation CAR-T cells were added another co-stimulatory molecule on the basis of the 2nd generation CAR (19). In order to obtain the best efficacy and the clinical application in the treatment of esophageal cancer in the future, we chose the 2 nd generation of the CAR modified T cells to study for tumor cell killing.

The key to treat ESCC with CAR-T cell immunotherapies is to prevent immune escape via targeting antigen on the membrane of carcinoma cells. EphA2 (erythropoietin-producing hepatocellular receptor A2), which is one of the Eph family, becomes known as a surface antigen (20). EphA2 overexpression brings about carcinogenic effects including oncogenesis, EMT transformation, vasculogenesis and cell growth $(21,22)$. The unique dual role of EphA2 in tumorigenesis and angiogenesis makes it one of the most attractive targets for tumor therapy (23). Recent study of EphA2.CAR-T cells immunotherapy indicated that they were able to recognize and clear out EphA2-postive glioma cells in vitro. By the way, leading to glioblastoma multiforme (GBM) tumor regression in vivo (20). In ESCC, it was reported that EphA2 overexpression was positive in 40 of the 80 patients (50\%) (24). Due to EphA2 is overexpressed in ESCC and EphA2 overexpression correlates with poor prognosis in ESCC (24) and EphA2 is a membrane antigen, a CAR targeting EphA2 could be the ideal solution for the immunotherapy of ESCC.

CAR-T immunotherapy for ESCC has not been reported so far. In the research, we devoted to construct a CAR specifically targeting EphA2 and transduct into T cell, and tested its cell killing efficacy in vitro. The research will open a new way for solid tumor treatment of ESCC by the second generation of EphA2.CAR-T cell immunotherapy in the future.

\section{Methods}

\section{Tissue specimens}

In this research, patients with ESCC were recruited in the Affiliated Hospital of Nantong University from 2010 to 2016. All diagnoses were on the basis of histopathological evidence. All patients were not received preoperative treatments for cancer, such as radiotherapy, chemotherapy or immunotherapy. Some fresh tissues (ESCC tissues and adjacent tissues) after surgical separation were immediately washed with sterile physiological saline before stored at $-80{ }^{\circ} \mathrm{C}$. Some tissues were fixed with $4 \%$ paraformaldehyde (PFA). The research was authorized by the Ethics Committee of the Affiliated Hospital of Nantong University (No. 2015-070). All patients agreed to use their tissues for scientific research.

\section{Immunobistochemistry}

The fixed specimens were dehydrated with graded alcohol, embedded with paraffin and cut into 5 - $\mu$ m-thick sections. The sections were implemented in sodium citrate solution $(\mathrm{pH}=6.0)$ and cooked at $100{ }^{\circ} \mathrm{C}$ for $10 \mathrm{~min}$ for antigen 
retrieval. Subsequently, the sections were placed in 3\% $\mathrm{H}_{2} \mathrm{O}_{2}$ for 30 min to eliminate endogenous peroxidases. The sections were reacted with mouse anti-human EphA2 primary antibody (1:200) (Santa Cruz Biotechnology, Dallas, TX) at $4{ }^{\circ} \mathrm{C}$ overnight. Then the pierce streptavidin poly-Horseradish peroxidase (ThermoFisher, Waltham, MA) was used to detect. The next day, some tissues were further incubated with rabbit anti-mouse IgG-TRITC secondary antibody (Millipore, Billerica, MA, USA) for $2 \mathrm{~h}$ at room temperature. The staining images were observed under a fluorescence microscope (Zeiss, Oberkochen, Germany).

\section{Cell culture}

ESCC cells ECA109 and TE-1 (Jennio Biotech Co.Ltd, Guangzhou, China) were cultured in DMEM media (Gibco, Carlsbad, CA) plus with 10\% FCS (fatal cattle serum) (lonsera, Shanghai, China) and $100 \mathrm{U} / \mathrm{mL}$ penicillinstreptomycin mixture (Solarbio, Beijing, China). EphA2. CAR-T cells and T cells were maintained in GT-T551 (Takara, Dalian, China) media plus with $10 \%$ FCS, 100 $\mathrm{U} / \mathrm{mL}$ penicillin-streptomycin mixture and 500 IU IL-2 (Novoprotein Scientific, Summit, NJ).

\section{Cell immunofluorescent staining}

Cells were cultured on glass coverslips (24-well plates) and fixed with $4 \%$ PFA for $20 \mathrm{~min}$ at $15-25^{\circ} \mathrm{C}$. Then the glass coverslips were washed $30 \mathrm{~min}$ with $0.01 \% \mathrm{PBS}$, and incubated with blocking solution containing $10 \%$ bovine serum albumin (BSA) for $2 \mathrm{~h}$ at $15-25^{\circ} \mathrm{C}$. After that, cells were reacted with mouse anti-human EphA2 primary antibody (1:200) (Santa Cruz Biotechnology, Dallas, TX) at $4{ }^{\circ} \mathrm{C}$ overnight. The next day, coverslips were allowed to incubate with rabbit anti-mouse IgG-TRITC secondary antibody (1:200) (Millipore, Billerica, MA) for $2 \mathrm{~h}$ at $15-25^{\circ} \mathrm{C}$. Finally, the cells were stained with Hoechst 33258 (Beyotime Institute of Biotechnology, Haimen, China) for $10 \mathrm{~min}$ and mounted with anti-fade solution, followed by examination under a fluorescence microscope (Zeiss, Oberkochen, Germany).

\section{Blood donor sample}

For all experiments, blood samples were collected with informed consent from healthy volunteers using protocol approved ethically by the Committee of Affiliated
Hospital of Nantong University. Based on operation declaration, lymphoprep density gradient centrifugation kit (STEMCELL Technologies, Vancouver, Canada) was adopted to isolate Peripheral Blood Mononuclear Cells (PBMCs) from blood.

\section{Construction of the second generation EphA2.CAR}

The EphA2-specific scFv was derived from the EphA2 monoclonal antibody (mAb) 4H5. GeneArt synthesized the codon-optimized gene, which was cloned into a lentivirus backbone containing the human $\mathrm{CD} 8 \alpha$ hinge and transmembrane domain, 4-1BB co-stimulatory domain and the CD3 -chain via 5' Xba I and 3' Bam H I sites. Another CAR containing the irrelevant scFv (FMC63) was served as control. Sequencing was used to verify the cloning of the EphA2.CAR and control.

\section{Transduction of $T$ cells and expansion}

Lentiviral supernatant was generated from 293T cells transfected with Lv-CAR-EphA2-GFP, pMD2G and pSPAX2. Human PBMCs which gained from healthy volunteer donors, were stimulated by CD28 antibodies $(1 \mathrm{ng} / \mathrm{mL})$ and OKT3 $(1 \mathrm{ng} / \mathrm{mL})$ (Novoprotein Scientific, Summit, NJ), and then cultured in media plus with $10 \%$ FCS, $100 \mathrm{U} / \mathrm{mL}$ penicillin-streptomycin mixture (Beyotime Institute of Biotechnology, Haimen, China) and IL-2 (500 IU/mL). T cells stimulated by OKT3/CD28 were then transduced with the constructed lentiviral vector which encoding EphA2.CAR on RetroNectin coated plates. Meanwhile, non-transduced $\mathrm{T}$ cells were used as negative control, and they were activated in the same culture condition. Transduced cells were cultured with IL-15 ( $5 \mathrm{ng} / \mathrm{mL})$ and IL-7 (100 ng/mL) for 14 to 21 days culture before subsequent analysis. The media and cytokines were changed every 3 days during culture or when passaging the $\mathrm{T}$ cells for splitting for expansion. In all the trials, we also compared the function of transduced and non-transduced $\mathrm{T}$ cells which obtained from the same donor.

\section{Flow cytometry (FCM)}

EphA2.CAR-T cells and none transduced T cells were collected and re-suspended by PBS plus $2 \%$ FCS. CAR-T cells transduction efficacies were assessed using FCM by detection of GFP autofluorescence. Data were analyzed by FlowJo 9.3.2 software. 


\section{Western blot}

Total proteins were isolated from ESCC tissues and cells by using RIPA buffer (Beyotime Institute of Biotechnology, Haimen, China), and quantified by BCA protein assay kit (Promega, Madison, WI, USA). Equivalent amount of proteins were transferred to PVDF membranes (Millipore, Billerica, MA, USA) after separated by SDS-PAGE. After blocking using $5 \%$ nonfat milk for $1 \mathrm{~h}$ at room temperature, membranes were reacted with mouse anti-human EphA2 primary antibody (1:500) (Santa Cruz Biotechnology, Dallas, TX, USA) in TBST plus with $5 \%$ nonfat milk at $4{ }^{\circ} \mathrm{C}$ overnight. After reaction with primary antibody, membranes were reacted with secondary HRP-conjugated goat antimouse antibody $(1: 1,000)$ (Santa Cruz Biotechnology, Dallas, TX, USA) for $2 \mathrm{~h}$ at room temperature. After washing, protein bands were measured by using an ECL assay kit (Pierce Protein Biology, Waltham, MA, USA) and imaged by chemiluminescence detection system (Tanon, Shanghai, China). The relative expression of EphA2 was determined as the ratio of gray scale value of EphA2 to $\beta$-actin. All experiments were repeated 3 times.

\section{Co-culture assays}

To examine the effect of $T$ cells and EphA2.CAR-T cells on ESCC cells killing in vitro, target cells (ECA109 and TE-1 cells) were seeded in triplicate in 96-well plate at a concentration of $1 \times 10^{4}$ cells per well. The both target cells were cultured together with $\mathrm{T}$ cells or EphA2.CAR-T cells at a serial of $\mathrm{E}$ : $\mathrm{T}$ (effector to target) ratio (1:1, 2:1, 5:1 and 10:1) for $24 \mathrm{~h}$ at $37^{\circ} \mathrm{C}$ and $5 \% \mathrm{CO}_{2}$. The morphology of the cells was observed under an inverted microscope (TS100, NIKON Instruments Inc, Japan) and photographed after co-culture.

\section{Crystal violet staining assay}

In order to measure the tumor cell killing effects of $\mathrm{T}$ cells and EphA2.CAR-T cells, crystal violet staining assay was used. ECA109 and TE-1 cells were seeded triplicate in 96well plate at a concentration of $1 \times 10^{4}$ cells per well, and co-cultured with $\mathrm{T}$ cells or EphA2.CAR-T cells at a serial of $\mathrm{E}$ : $\mathrm{T}$ ratio $(1: 1,2: 1,5: 1$ and $10: 1)$ for $24 \mathrm{~h}$ at $37^{\circ} \mathrm{C}$ and $5 \% \mathrm{CO}_{2}$. The supernatant of the culture medium were discarded, and each well was washed 2 times by $0.01 \%$ PBS. Cells were fixed with $4 \%$ PFA for $15 \mathrm{~min}$, and after that washed with $0.01 \%$ PBS for 3 times. Finally cells in each well were stained by crystal violet staining solution (Solarbio, Beijing, China). The images were observed under an inverted microscope (TS100, NIKON Instruments Inc, Japan) and photographed after staining.

\section{LDH cytotoxicity assay}

To assess the cytotoxic activity of EphA2.CAR-T cells, LDH release assay was used. Briefly, ECA109 and TE-1 cells were seeded triplicate in 96-well plate at a concentration of $1 \times 10^{4}$ cells per well, and co-cultured with $\mathrm{T}$ cells or EphA2.CAR-T cells at a serial of E: T ratio $(1: 1,2: 1,5: 1$ and $10: 1)$ for $24 \mathrm{~h}$ at $37{ }^{\circ} \mathrm{C}$ and $5 \% \mathrm{CO}_{2}$. The supernatants were collected after co-culture to analyze the production of LDH by using of a $\mathrm{LDH}$ release assay kit (Beyotime Institute of Biotechnology, Haimen, China) according to the operation declaration. OD (optical density) values in each well were read at $490 \mathrm{~nm}$ using the multimode reader (Thermo Electron, Waltham, MA). The $\mathrm{LDH}$ release activity was analyzed by the following formula: $\left(\mathrm{OD}_{\text {sample }}-\mathrm{OD}_{\text {blank }}\right) /\left(\mathrm{OD}_{\text {maximum release }}-\mathrm{OD}_{\text {blank }}\right) \times 100 \%$.

\section{Analysis of cytokine production}

To measure the production of cytokines in vitro, ECA109 and TE- 1 cells were also seeded triplicate in 96-well plate at a concentration of $1 \times 10^{4}$ cells per well, and co-cultured with $\mathrm{T}$ cells or EphA2.CAR-T cells at E: T ratio $(5: 1)$ for $24 \mathrm{~h}$ at $37{ }^{\circ} \mathrm{C}$ and $5 \% \mathrm{CO}_{2}$. Supernatants were collected after coculture and the levels of TNF- $\alpha$ and IFN- $\gamma$ were analyzed by ELISA kit (Jiancheng, Nanjing City, China) follow the instructions. The OD values were tested at $450 \mathrm{~nm}$ by the multimode reader (Thermo Electron, Waltham, MA, USA).

\section{Statistical analysis}

The quantitative date was showed as means \pm standard deviation (SD). Student's $t$-test was used to analyze the difference. $\mathrm{P}<0.05$ was regarded as the level of significant in all of the analyses.

\section{Results}

\section{EphA2 is expressed in ESCC}

Initially, we detected EphA2 expression in ESCC tissues and adjacent tissues by using immunofluorescence and immunohistochemistry staining. It was observed obviously 
that the expression of EphA2 on the membrane of ESCC cell and its expression were increased in ESCC tissues, compared with adjacent tissues. It was showed in Figure 1A,B. Secondly, we detected EphA2 expression in ESCC cell line ECA109 and TE-1 by cell immunofluorescence. It was also observed obviously that the expression of EphA2 on the membrane of ECA109 and TE-1 cells. It was showed in Figure 1C. Thirdly, we detected the expression of EphA2 in ESCC tissues and cells by Western blot. As anticipated, the expressions of EphA2 were significantly increased in ESCC tissues and ESCC cells, compared to adjacent tissues (Figure 1D) $(\mathrm{P}<0.05)$. It was showed in Figure 1D. These results confirmed that EphA2 expression was significantly upregulated in ESCC tissues and cells, and EphA2 could be served as TAA of CAR for ESCC treatment.

\section{Construction of the second generation EphA2.CAR T cells}

The schematic diagrams of construction of EphA2.CAR and CD19.CAR were showed in Figure 2A. CAR-T cells transduction efficacies were assessed using FCM by detection of GFP autofluorescence. The results of FCM analysis showed that the positive percentages of GFP in EphA.CAR group and CD19.CAR group were 57.6\% and $61.4 \%$. It was showed in Figure $2 B$. Compared to the activated $\mathrm{T}$ group, the positive percentages of GFP in CAR group were significantly higher.

\section{EphA2.CAR-T cells caused ESCC cell killing in vitro}

Firstly, co-culture experiment was used to illustrate the effect of cell killing of EphA2.CAR-T cells on ECA109 and TE-1 cells in vitro. The T cells and EphA2.CAR-T cells were observed dose-dependent cell killing after co-culture with target ESCC ECA109 and TE-1 cells at E: T ratio of 1:1 to $10: 1$ for $24 \mathrm{~h}$ under an inverted microscope. When the E: T ratio was 1:1, EphA2.CAR-T cells could eliminate part of ECA109 and TE-1 cells. It was amazing that almost all ECA109 and TE-1 cells were cleared when the E: T ratio reached to 5:1. The ability of cell killing of EphA2. CAR-T cells on ECA109 and TE- 1 cells was better than that of T cells. It was showed in Figure 3 A.

Secondly, crystalline violet staining assay was used to confirm the efficacy of cell killing of EphA2.CAR-T cells on ESCC cells. The results were similar to the observed results under an inverted microscope. It was also showed that the $\mathrm{T}$ cells and EphA2.CAR-T cells both demonstrated the dose-dependent cell killing. It was showed in Figure $3 B$.
Thirdly, the cytotoxic activity of EphA2.CAR-T cells for ESCC cells was analyzed by LDH release assay kit. As anticipated, the LDH release in EphA2.CAR-T cells group was significantly increased, compared to the non-transduced $\mathrm{T}$ cells group $(\mathrm{P}<0.01)$. And, the effect of EphA2.CAR-T cells on the LDH release from target ESCC cells ECA109 and TE- 1 was also dose-dependent. It was shown in Figure $3 C$. The above results revealed that the EphA2. CAR-T cells had good effects on the target ESCC cells killing.

\section{EphA2.CAR-T cells induce cytokines release}

Cytokines are small molecule peptides, secreted by immune cells, could regulate the cell function (such as cell division and differentiation, immune function and inflammation, etc.). So, we tested the production of cytokines by EphA2. CAR-T cells after co-culture with ESCC cells by ELISA. The results of ELISA assay showed that the levels of TNF- $\alpha$ and IFN- $\gamma$ in EphA2.CAR-T cells were notably raised, compared with non-transduced $\mathrm{T}$ cells $(\mathrm{P}<0.05)$. The secreted TNF- $\alpha$ in EphA2.CAR-T cells after coculture with ECA109 and TE-1 cell was increased over 2 -fold than $\mathrm{T}$ cells $(\mathrm{P}<0.01)$. It was showed in Figure $4 A$. The secreted IFN- $\gamma$ in EphA2.CAR-T cells after co-culture with ECA109 was increased over 3 -fold $(\mathrm{P}<0.01)$, however IFN- $\gamma$ was increased only over 2 -fold after co-culture with TE- 1 cells $(\mathrm{P}<0.05)$. It was showed in Figure $4 B$. These results indicated that EphA2.CAR-T cells effectively promoted the release of TNF- $\alpha$ and IFN- $\gamma$.

\section{Discussion}

The development of CAR T technology has brought good news to the treatment of cancer patients, special for leukemia (25). In 2017, a CAR-T cell therapy, Kymriah (TM) (CTL019) by Novartis, for B-cell acute lymphatic leukemia (ALL) was ratified by US food \& drug administration (FDA) (26). It was the first FDA approval for CAR-T cell treatment and encouraging researchers to develop various CAR-T cells for immunotherapy in the future. In this study, we had first successfully constructed the second generation EphA2.CAR and established the EphA2.CAR-T cells. We indicated that EphA2.CAR-T cells could kill ESCC lines ECA109 and TE-1 effectively, and promote significantly the release of cytokines.

Tumor immunotherapy is a treatment method for the prevention and treatment of malignant tumors by 

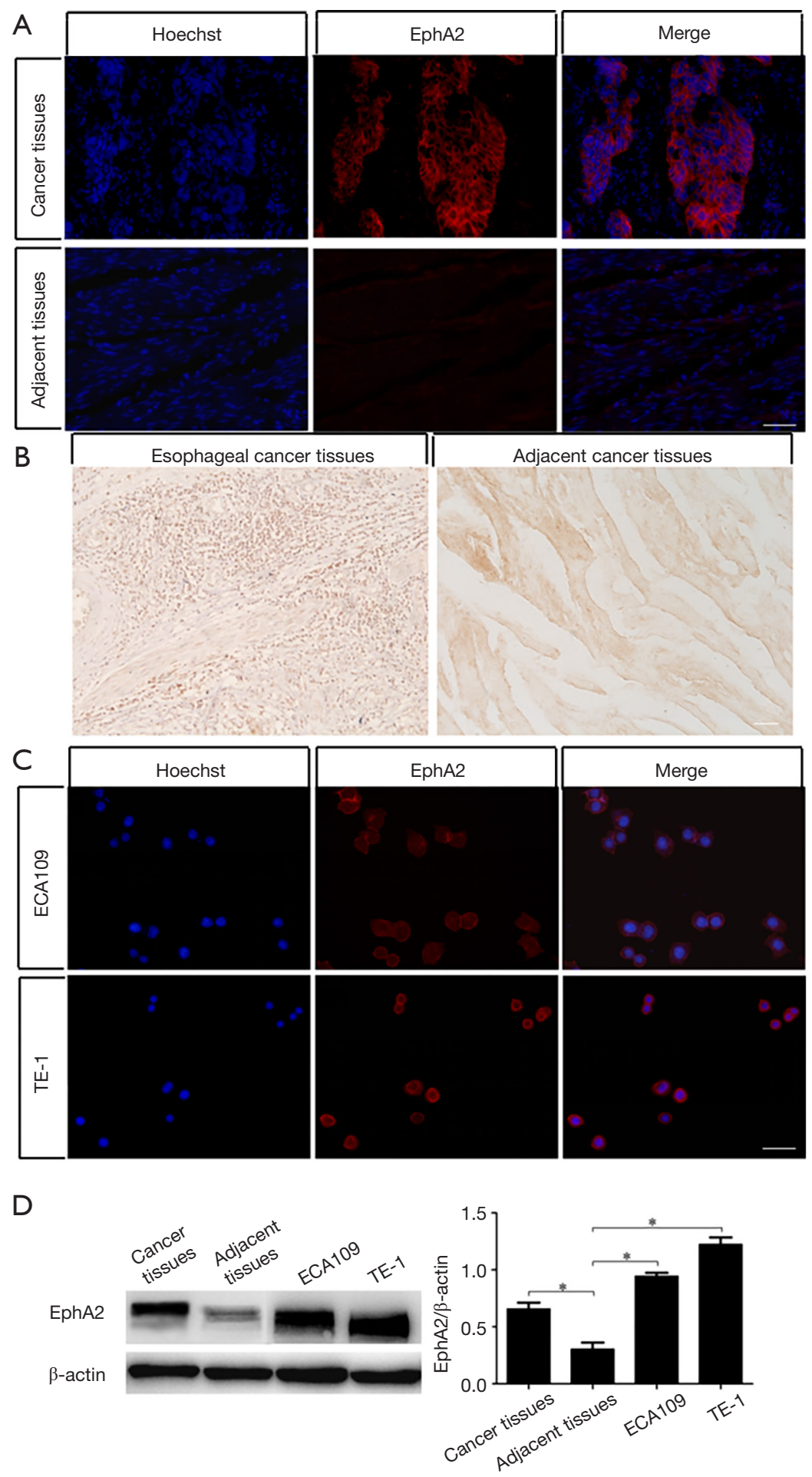

Figure 1 The expression of EphA2 in ESCC tissues and cells. (A) Cellular immunofluorescence staining of EphA2 in ESCC cancer tissues and adjacent tissues; (B) immunohistochemistry staining analysis EphA2 in ESCC cancer tissues and adjacent tissues; (C) cellular immunofluorescence staining of EphA2 in ESCC cell lines ECA109 and TE-1; (D) western blot analysis of EphA2 in ESCC cancer tissues, adjacent tissues, ECA109 and TE-1 cells. Bar $=50 \mu \mathrm{m} .{ }^{*}, \mathrm{P}<0.05$. 

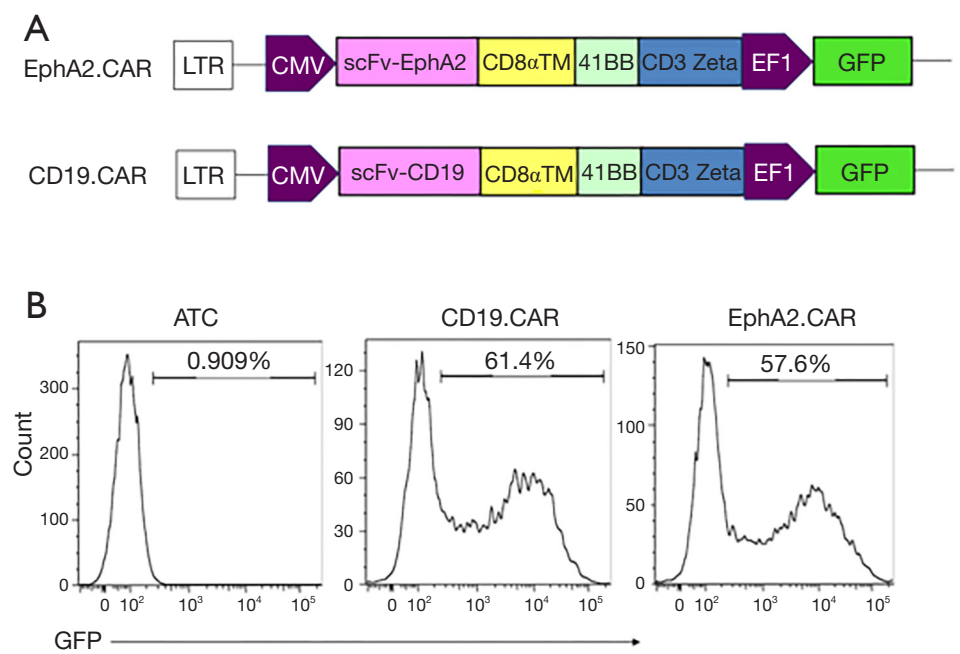

Figure 2 The construction of the second generation EphA2.CAR-T cells. (A) The EphA2.CAR was generated by the cloning a single chain variable fragment derived from the EphA2 monoclonal antibody a CD8 $\alpha$ hinge and transmembrane domain, a 4-1BB co-stimulatory domain and a CD3Zeta activation domain into a Lentiviral backbone; (B) EphA2.CAR expression was measured by fluorescence activated cell sorting analysis. ATC, activated T cells; TM, transmembrane region.
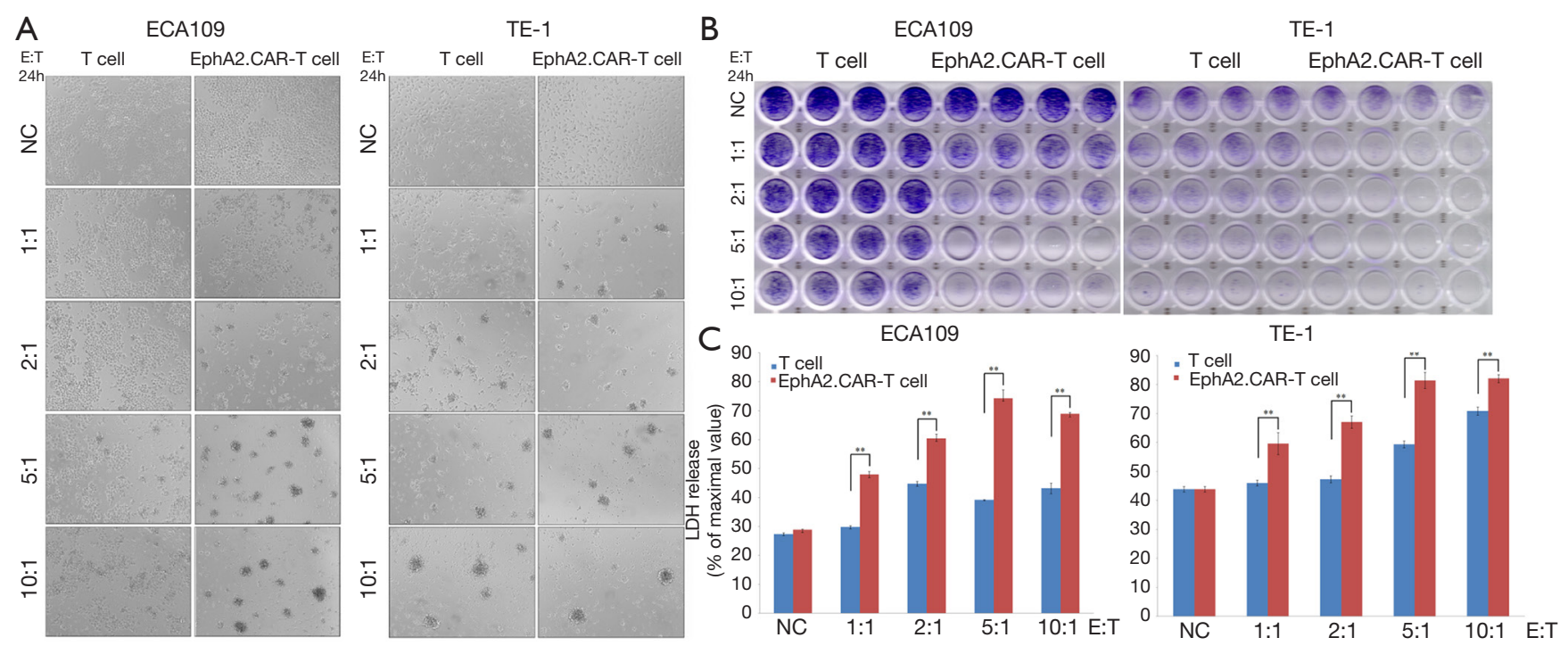

Figure 3 Detection of efficacy of EphA2.CAR-T cells in ESCC cell lines ECA109 and TE-1. The effects of EphA2.CAR-T cells and nontransduced T-cells in killing the ECA109 and TE-1 cells was observed by inverted microscope and measured by Crystal violet staining assay. (A) The image of the ECA109 and TE-1 co-cultured with EphA2.CAR T cells and non-transduced T-cells with different E: T ratio using the inverted microscope; (B) the photograph of crystalline violet staining assay of the ECA109 and TE-1 co-cultured with EphA2.CAR-T cells and non-transduced T-cells with different E: T ratio; $(\mathrm{C})$ detection of the LDH release in the supernatant of the ECA109 and TE-1 co-cultured with EphA2.CAR-T cells and non-transduced T-cells with different E: T ratio after 24 h. *, $\mathrm{P}<0.05$. 

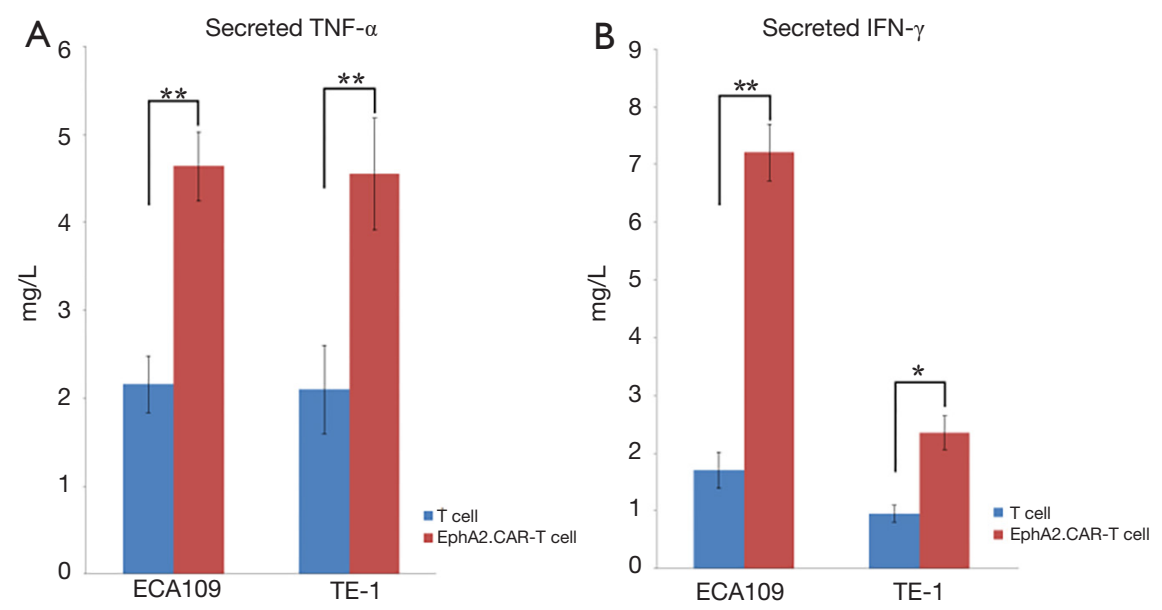

Figure 4 Detection of tumor necrosis factor- $\alpha(\mathrm{TNF}-\alpha)$ and interferon-gamma (IFN- $\gamma)$. TNF- $\alpha$ and IFN- $\gamma$ secreted in the supernatant of the ECA109 and TE-1 co-cultured with EphA2.CAR-T cells and non-transduced T-cells with different E: T ratio after 24 h was analyzed by ELISA. *, $\mathrm{P}<0.05 ;{ }^{* *}, \mathrm{P}<0.01$.

regulating the body's immune status and achieve (27). Cytokines, tumor vaccine, immune checkpoint blockade immunotherapeutic agent (28) and adoptive cell therapy (ACT) has been represented in clinical application and demonstrated great clinical curative effects. The technology of ACT was transferring autologous or allogeneic immune cells to patients after these cells amplification in vitro. The transferred immune cells could directly kill tumor cells and mobilize the body immune function.

The current commonly used anti-tumor effector cells could be divided into two categories: the first category was nonspecific effector cell, including autologous lymphokine activated killer cells, cytokine induced killer cells (29) and natural killer cells (30), another type was specific effector cells, including CAR-T cell. The basic characteristics of CAR-T cell was cell killing specifically via recognizing specific TAA $(31,32)$.

EphA2 overexpression induces carcinogenic effects including oncogenesis (33), EMT transformation (34), vasculogenesis (23) and cell growth (35). The unique dual role of EphA2 in tumorigenesis and angiogenesis made it one of the most attractive targets for tumor therapy. And it was reported that EphA2.CAR-T cells were able to recognize and kill EphA2-postive glioma cells in vitro and induced GBM tumor regression in vivo (20). So, at first, we detected EphA2 expression in ESCC tissues and adjacent tissues via immunofluorescence and immunohistochemistry staining. These results proved that EphA2 expression was up-regulated in ESCC tissues and expressed on the membrane of ESCC cells. Therefore, EphA2 could be served as TAA of CAR for ESCC treatment.

After the successful construction of EphA.CAR, we firstly considered the tumor cells killing by EphA.CAR-T cells using co-culture with target ECA109 and TE-1 cells in vitro. The T cells and EphA2.CAR-T cells were observed as dose-dependent cell killing at E:T ratio of 1:1 to 10:1 for 24 $\mathrm{h}$ under an inverted microscope. The efficacy of cell killing of EphA2.CAR-T cells on ECA109 and TE-1 cells was better than that of $\mathrm{T}$ cells. Crystalline violet staining assay and $\mathrm{LDH}$ release assay was applied to further confirm the cell killing of EphA2.CAR-T cells. The both results were similar to the result observed under an inverted microscope. It was concluded that EphA2.CAR-T cells demonstrated a dose-dependent and specific cell killing in vitro.

TNF- $\alpha$ and IFN- $\gamma$ are cytokines both with anti-tumor characteristics $(36,37)$. The production of TNF- $\alpha$ and IFN- $\gamma$ by EphA2.CAR-T cells after co-culture with ESCC cells was tested by ELISA. The release levels of TNF- $\alpha$ and IFN- $\gamma$ in EphA2.CAR-T cells were notably raised. EphA2. CAR-T cells played a vital role in cell killing via increasing cytokines release. The successful cloning of EphA2 scFv into the CAR to the antigen could activate the intracellular signaling transduction of $\mathrm{T}$ cells to produce cytokines TNF- $\alpha$ and IFN- $\gamma$.

In summary, we firstly successfully constructed the second generation of EphA2.CAR and established EphA2. CAR-T cells. The EphA2.CAR-T cells showed the better efficacy of ESCC cell killing and production of cytokines 
in vitro. But, there was a limitation that there was no data of clinical cases or in vivo examination in this study. Next, we will test the effect of anti-tumor of EphA2.CAR-T cells in vivo and prepare for clinical trial. These findings open a new way for treatment of ESCC by immunotherapy in the future.

\section{Acknowledgements}

Funding: This study was supported by grants from the National Natural Science Foundation of China (81101159 and 81502055), the Natural Science Foundation of Jiangsu Province, China (BK20151268 and BK20151347), and Health Project of Jiangsu Province (Z201503).

\section{Footnote}

Conflicts of Interest: The authors have no conflicts of interest to declare.

Ethical Statement: The research was authorized by the Ethics Committee of the Affiliated Hospital of Nantong University (No. 2015-070). All patients agreed to use their tissues for scientific research.

\section{References}

1. Ferlay J, Soerjomataram I, Dikshit R, et al. Cancer incidence and mortality worldwide: sources, methods and major patterns in GLOBOCAN 2012. Int J Cancer 2015;136:E359-86.

2. Pennathur A, Gibson MK, Jobe BA, et al. Oesophageal carcinoma. Lancet 2013;381:400-12.

3. Ohashi S, Miyamoto S, Kikuchi O, et al. Recent advances from basic and clinical studies of esophageal squamous cell carcinoma. Gastroenterology 2015;149:1700-15.

4. Tran E, Robbins PF, Rosenberg SA. 'Final common pathway' of human cancer immunotherapy: targeting random somatic mutations. Nat Immunol 2017;18:255-62.

5. Rosenberg SA, Restifo NP. Adoptive cell transfer as personalized immunotherapy for human cancer. Science 2015;348:62-8.

6. Topalian SL, Wolchok JD, Chan TA, et al. Immunotherapy: The path to win the war on cancer? Cell 2015;161:185-6.

7. Cheadle EJ, Gornall H, Baldan V, et al. CAR T cells: driving the road from the laboratory to the clinic. Immunol Rev 2014;257:91-106.
8. Liu L, Sommermeyer D, Cabanov A, et al. Inclusion of Strep-tag II in design of antigen receptors for T-cell immunotherapy. Nat Biotechnol 2016;34:430-4.

9. Lim WA, June CH. The Principles of Engineering Immune Cells to Treat Cancer. Cell 2017;168:724-40.

10. Wu Y, Jiang $M$. The revolution of lung cancer treatment: from vaccines, to immune checkpoint inhibitors, to chimeric antigen receptor T therapy. Biotarget 2017;1:7.

11. Zhukovsky EA, Morse RJ, Maus MV. Bispecific antibodies and CARs: generalized immunotherapeutics harnessing $\mathrm{T}$ cell redirection. Curr Opin Immunol 2016;40:24-35.

12. Yu S, Li A, Liu Q, et al. Chimeric antigen receptor $\mathrm{T}$ cells: a novel therapy for solid tumors. J Hematol Oncol 2017;10:78.

13. Srivastava S, Riddell SR. Engineering CAR-T cells: Design concepts. Trends Immunol 2015;36:494-502.

14. Lin J, Yang L, Silva HM, et al. Increased generation of Foxp3(+) regulatory $\mathrm{T}$ cells by manipulating antigen presentation in the thymus. Nat Commun 2016;7:10562.

15. Enblad G, Karlsson H, Loskog AS. CAR T-Cell Therapy: The Role of Physical Barriers and Immunosuppression in Lymphoma. Hum Gene Ther 2015;26:498-505.

16. Jensen MC, Riddell SR. Design and implementation of adoptive therapy with chimeric antigen receptor-modified T cells. Immunol Rev 2014;257:127-44.

17. Song DG, Ye Q, Poussin M, et al. CD27 costimulation augments the survival and antitumor activity of redirected human T cells in vivo. Blood 2012;119:696-706.

18. Bai W, Wang P, Yu F. CAR-T cells shed light on the treatments of fatal liver diseases. Biotarget 2018;2:6.

19. Carpenito C, Milone MC, Hassan R, et al. Control of large, established tumor xenografts with genetically retargeted human T cells containing CD28 and CD137 domains. Proc Natl Acad Sci U S A 2009;106:3360-5.

20. Chow KK, Naik S, Kakarla S, et al. T cells redirected to EphA2 for the immunotherapy of glioblastoma. Mol Ther 2013;21:629-37.

21. Miao H, Gale NW, Guo H, et al. EphA2 promotes infiltrative invasion of glioma stem cells in vivo through cross-talk with Akt and regulates stem cell properties. Oncogene 2015;34:558-67.

22. Kaushansky A, Douglass AN, Arang N, et al. Malaria parasites target the hepatocyte receptor EphA2 for successful host infection. Science 2015;350:1089-92.

23. Neill T, Buraschi S, Goyal A, et al. EphA2 is a functional receptor for the growth factor progranulin. J Cell Biol 2016;215:687-703.

24. Miyazaki T, Kato H, Fukuchi M, et al. EphA2 
overexpression correlates with poor prognosis in esophageal squamous cell carcinoma. Int J Cancer 2003;103:657-63.

25. Rodgers DT, Mazagova M, Hampton EN, et al. Switchmediated activation and retargeting of CAR-T cells for B-cell malignancies. Proc Natl Acad Sci U S A 2016;113:E459-68.

26. Singh N, Shi J, June CH, et al. Genome-Editing Technologies in Adoptive T cell immunotherapy for cancer. Curr Hematol Malig Rep 2017;12:522-9.

27. Lichty BD, Breitbach CJ, Stojdl DF, et al. Going viral with cancer immunotherapy. Nat Rev Cancer 2014;14:559-67.

28. Romano E, Romero P. The therapeutic promise of disrupting the PD-1/PD-L1 immune checkpoint in cancer: unleashing the CD8 $\mathrm{T}$ cell mediated anti-tumor activity results in significant, unprecedented clinical efficacy in various solid tumors. J Immunother Cancer 2015;3:15.

29. Gao X, Mi Y, Guo N, et al. Cytokine-Induced Killer Cells As Pharmacological Tools for Cancer Immunotherapy. Front Immunol 2017;8:774.

30. Peng H, Tian Z. Natural Killer Cell Memory: Progress and Implications. Front Immunol 2017;8:1143.

31. Glienke W, Esser R, Priesner C, et al. Advantages and

Cite this article as: Shi H, Yu F, Mao Y, Ju Q, Wu Y, Bai W, Wang P, Xu R, Jiang M, Shi J. EphA2 chimeric antigen receptor-modified $\mathrm{T}$ cells for the immunotherapy of esophageal squamous cell carcinoma. J Thorac Dis 2018;10(5):2779-2788. doi: $10.21037 /$ jtd.2018.04.91 applications of CAR-expressing natural killer cells. Front Pharmacol 2015;6:21.

32. Hinrichs CS, Restifo NP. Reassessing target antigens for adoptive T-cell therapy. Nat Biotechnol 2013;31:9991008.

33. Youngblood VM, Kim LC, Edwards DN, et al. The Ephrin-A1/EPHA2 Signaling Axis Regulates Glutamine Metabolism in HER2-Positive Breast Cancer. Cancer Res 2016;76:1825-36.

34. Dunne PD, Dasgupta S, Blayney JK, et al. EphA2 Expression Is a Key Driver of Migration and Invasion and a Poor Prognostic Marker in Colorectal Cancer. Clin Cancer Res 2016;22:230-42.

35. Takasugi M, Okada R, Takahashi A, et al. Small extracellular vesicles secreted from senescent cells promote cancer cell proliferation through EphA2. Nat Commun 2017;8:15729.

36. Kalliolias GD, Ivashkiv LB. TNF biology, pathogenic mechanisms and emerging therapeutic strategies. Nat Rev Rheumatol 2016;12:49-62.

37. Lasfar A, Zloza A, de la Torre A, et al. IFN-lambda: A New Inducer of Local Immunity against Cancer and Infections. Front Immunol 2016;7:598. 